\section{Genetische Beratung}

\section{J. Arnemann}

Abteilung Molekulargenetik, Labor Dr. Wisplinghoff, Köln, Deutschland

Synonym(e) Humangenetische Beratung

Englischer Begriff genetic counselling

Definition Eine humangenetische Beratung, oftmals nur genetische Beratung genannt, ist eine medizinische Dienstleistung, die von Fachärzten für Humangenetik oder anderen Fachärzten mit einer entsprechenden Zusatzqualifikation für Ratsuchende oder Patienten mit Fragen zu Fehlbildungen, Behinderungen oder genetisch bedingten Erkrankungen angeboten wird.

Beschreibung Die genetische Beratung behandelt Fragen zu erblich bedingten Syndromen und Erkrankungen. Durch eine intensive Anamneseerhebung der gesundheitlichen Vorgeschichte des Ratsuchenden oder Patienten und der Familien- angehörigen bis zur großelterlichen Generation werden zunächst die Basisinformationen gesammelt, die Grundlagen für eine genetische Risikoabschätzung und das Wiederholungsrisiko sind. Es werden weiterhin detaillierte Informationen zu den Krankheiten gegeben und bei Kinderwunsch Möglichkeiten einer vorgeburtlichen Diagnostik vorgestellt. Bei genetisch bedingten Krebserkrankungen kann eine interdisziplinäre Vorgehensweise mit anderen Fachärzten organisiert werden.

Bei unklaren Krankheitsbildern wird versucht durch ausführliche Literatur- und Datenbankrecherchen eine diagnostische Zuordnung vorzunehmen.

Die humangenetische Beratung sollte immer nicht direktiv erfolgen, d. h., der Berater versucht, Tatbestände und mögliche Konsequenzen objektiv darzustellen, ohne eine persönliche Wertung vorzunehmen.

\section{Literatur}

Harper PS (2010) Practical genetic counselling, 7. Aufl. Taylor \& Francis, London 